\section{P526 \\ EMOTIONAL AND PERSONAL FEATURES OF ADOLESCENTS WITH OVERWEIGHT AND OBESITY}

Lyubov Rychkova, Vladimir Polyakov, Aryuna Kosovtseva*. Scientific Centre for Family Health and Human Reproduction Problems, Irkutsk, Russian Federation

10.1136/archdischild-2019-epa.861

Aim To identify the most significant emotional and personal features of adolescents with overweight and obesity.

Methods The analysis included 279 subjects: 198 adolescents with normal body weight, 81 - with overweight and obesity. The study was conducted from January 2015 to April 2016 in the rural areas of the Republic of Buryatia (Russia). Evaluation of the growth and weight parameters of adolescents was carried out using reference WHO values by AnthroPlus calculator. The body mass index (BMI) was calculated as weight $(\mathrm{kg})$ divided by height $(\mathrm{m})^{2}$. Body weight was considered excessive with a BMI $>85$ th percentile of distribution for a given sex and age, obesity was established with BMI $>95$ th percentile. Each adolescent was asked to complete the following questionnaires: The State-Trait Anxiety Inventory (STAI) Russian version, Buss-Durkee Hostility Inventory (BDHI), reduced Minnesota Multiphasic Personality Inventory (MiniMMPI), Pediatric Quality of Life Inventory (PedsQL) 4.0 Generic Core Scales.

Results Significant differences were found only in the MiniMMPI questionnaire. The values of Mini-MMPI 5 scale (sentimentality, sensitivity, vulnerability) are significantly lower in adolescents with overweight and obesity compared to adolescents with normal body weight (1.13 and 2.57, respectively ( $\mathrm{P}$ $=0.001)$. Similar data were found in relation to the MiniMMPI 0 scale (introversion) (1.06 and 1.74 , respectively $(\mathrm{P}=$ $0.021)$. In both groups the values do not exceed the normative level and are not an accentuation of the character.

These personal features of adolescents with overweight and obesity are manifested in greater extroversion, sociability, openness, and activity of personal position. This adolescents are less inclined to 'withdraw into themselves' in a situation of stress, choosing a different way to control negative emotions. Such adolescents are outwardly less vulnerable, able to quickly switch from a negative situation. Adolescents with normal body weight perceive emotions differently. They are more closed and susceptible to the environment and negative situations.

Conclusion Adolescents with overweight and obesity are more extraverted and less sensitive. The comprehension of the association of extroversion and obesity is consisted in the regulation of excitement in extroverts. Extroverts further increase the level of arousal through food intake. In addition, extroverts are characterized by external type of eating behavior. The external attractiveness of food and other sensory eating signals are a stimulus to the consumption of food.

\section{P527 A FRAMEWORK DOCUMENT TO FACILITATE HEALTHY WEIGHT FOR CHILDREN AGED 0-6 YEARS IN IRELAND}

${ }^{1}$ Phil Jennings*, ${ }^{2}$ Fionnuala Cooney. 'Department of Public - HSE, Tullamore, Ireland; ${ }^{2}$ Department of Public - HSE, Dublin, Ireland

10.1136/archdischild-2019-epa.862

Internationally, obesity is recognised as a major public health challenge. Currently in Ireland we have a significant problem with $19 \%$ of 3 year olds overweight, with a further $5 \%$ obese. Among 5 year olds, 15\% are overweight and 5\% obese. There is a social gradient evident with prevalence of overweight and obesity being 6\%-7\% higher in schools in disadvantaged areas. The adverse consequences of childhood obesity can be lifelong and intergenerational. The total lifetime costs of childhood obesity in the Republic of Ireland are estimated to be $€ 4.6$ billion. A reduction of the body mass index (BMI) by just $5 \%$ in our population has been estimated to reduce the lifetime cost of childhood overweight and obesity by $€ 1.1$ billion. A Healthy Weight for Ireland Obesity Policy and Action Plan 2016-2025, has set a target of a $0.5 \%$ per annum reduction in excess weight in children and a $10 \%$ closing of the gap between socio-economic groups.

A multidisciplinary Healthy Weight for Children (HWfC) working group has been convened by the Health Service Executive (HSE) with representatives from the Healthy Childhood programme, the Healthy Eating Active Living programme, TUSLA/the Child and Family Agency, safefood and academia. Using international and local evidence, this group has informed and supported the development of a framework document with the purpose of providing a strategic direction to facilitate healthy weight among children in Ireland with a strong focus is on prevention among 0-6 year olds, inclusive of the preconception and antenatal periods.

A portfolio of recommendations is presented under 10 defined areas for action. Central to the process is the need for a focus on a population level approach in prevention with a coherent, consistent and equitable process that is informed and quality assured with an inbuilt evaluation and monitoring system. Interventions for healthy behaviours are to be targeted at critical periods in the life-course, especially during interactions with services in the healthcare, childcare, social care and education sectors. Additional supportive interventions are necessary in the areas of legislation, mass communications/social marketing, community-based interventions and supplementary targeted supports for those most in need. Leadership, governance, monitoring and evaluation are identified as crucial for successful implementation.

This framework document is underpinned by the principle that government and society have a moral and legal responsibility to act on behalf, and in the best interests of children in reducing the risk of obesity through protecting children's rights to health.

\section{P528 LIPID METABOLISM, INSÜLIN RESISTANCE AND NAFLD IN OBESITY}

IIlhan Hazer, Hilmi Onur Kabukçu, Murat Yağcı, Zeynep Ertürk, Gonca Kllıç Yıldırım, Birgül Kirel*. Eskişehir Osmangazi University, Faculty of Medicine, Department of Pediatric Endocrinology, Eskişehir, Turkey

\subsection{6/archdischild-2019-epa.863}

Obesity, insülin resistance (IR) and non-alcoholic fatty liver disease (NAFLD) are the major components of the metabolic syndrome. On the other hand, it is known that primer hypolipidemia is associated with increased prevalence of NAFLD.

We evaluated the associations of IR and NAFLD with lipid metabolism parameters in 357 obese children and adeloscents (199 girls, 158 boys) from their hospital records. The LDL-C levels were compared to the reference LDL-C levels according to age and sex. The patients were divided into three groups 
according to the serum levels of LDL-C; Groups 1, 2, 3 consisted of hypolipidemic patients $(n=61)$, normolipidemic patients $(n=135)$ and hyperlipidemic patients $(n=161)$, respectively. HOMA-IR above 3.42 was defined as IR.

Body mass index were not different among these groups $(\mathrm{p}$ $>0.05)$. The frequency of NAFLD was $44.5 \%$ in the entire study group and were $52 \%, 44.7 \%$ and $41.4 \%$ in the Groups 1,2 , 3, respectively. The frequency of NAFLD were not different among these three groups ( $p>0.05$ ). In NAFLD patients, HDL-C levels were lower than in patients without NAFLD $(p>0.01)$ and negatively correlated with ALT levels $(\mathrm{r}=-0.1, \mathrm{p}<0.05)$.

In patients with IR $(n=172)$, the frequency of NAFLD and triglyceride levels were higher and HDL-C levels were lower than in patients without IR $(n=178)$. The frequency of IR were not different among the Groups 1,2 and 3 ( $\mathrm{p}>0.05$ ).

It is found out that hypolipidemic obese patients, clinically not given much attention, have NAFLD at least as frequent as hyperlipidemic patients do. On the other hand, obese patients with IR and NAFLD have atherogenic profile; high trygliyceride and low HDL-C levels. Low HDL-C levels may be related with also hepatosteotitis in the NAFLD. Therefore, abnormalities in lipid metabolism are significant contributers of obesity and associated co-morbidities.

\section{P529 RECOGNITION OF OBESITY IN CHILDREN ATTENDING A PAEDIATRIC EMERGENCY DEPARTMENT (THE ROIC STUDY)}

Jeanne Cloonan*, Emer Dwyer, Eimear Carlos, Ireti Farombi. Our Lady of Lourdes Hospital, Drogheda, Ireland

\subsection{6/archdischild-2019-epa.864}

Background Childhood obesity is a global public health problem, linked to adult obesity and its associated co-morbidities. A study in a Paediatric Emergency Department in London in the BMJ in 2014 found a low recognition and referral rate for Paediatric Obesity. ${ }^{1}$ However, no such studies have been performed to date in Ireland.

Aims To ascertain the incidence of obesity in patients attending our Paediatric Emergency Department.

To establish recognition of obesity by paediatric medical staff.

Methods This was a Prospective Study. Nursing staff were asked to measure heights along with weights at triage of children between 2 and 16 years of age, over a one week period. Inclusion criteria were paediatric medical patients, aged 2-16 yrs. Surgical/trauma patients, those who's height had not been documented and those who did not wait to be seen were excluded.

BMI centiles were calculated on all those meeting inclusion criteria. In those who plotted as overweight or obese, age, gender, presenting complaint, co-morbidities and nutritional history, if taken, were documented, along with whether the raised BMI was recognised/documented by medical staff and if they received a referral for further follow-up.

Results A search of the Emergency Department IPMS system found 222 patients aged 2-16 years attended the Paediatric Emergency Department during the week of the study.

The ED charts of 149 patients who met criteria were reviewed. Heights were recorded in 61/149 (40\%). BMI centiles were plotted for those patients and 20/61 (33\%) were found to be overweight or obese.

Of these patients, 10 were overweight (between $91^{\text {st }}$ and $98^{\text {th }}$ Centile) and 10 were obese ( $>98^{\text {th }}$ Centile). $1 / 20$ patients $(5 \%)$ was noted to be obese and was referred to a dietician. $12(60 \%)$ were male and $8(40 \%)$ were female.

The most common presenting complaints were abdominal pain $4(20 \%)$ and chest pain $3(15 \%)$.

$5 / 20(25 \%)$ had other documented co-morbidities.

Conclusions These results show a low recognition of paediatric obesity in the Emergency Department by paediatric medical staff.

These results also show a high incidence of obesity in our population however as only $40 \%$ of patients had heights measured the true incidence is difficult to determine.

These results support the need for further education of paediatric staff in the recognition of obesity and the development of guidelines and an appropriate referral pathway.

\section{REFERENCE}

1. Knight M, Booth C. Obesity management in a Paediatric Emergency Department BMJ Qual Improv Rep. 2014;3(1):u203067.w1454.

\section{P530 ASSOCIATIONS BETWEEN DISORDERED EATING ATTITUTES AND BEHAVIORS IN ADOLESCENTS WITH OBESITY AND DEPRESSION AND ANXIETY}

${ }^{1}$ Aslı Okbay Güneş*, ${ }^{2}$ Müjgan Alikaşifoğlu, ${ }^{3}$ Ezgi Şen Demirdöğen, ${ }^{4}$ Ethem Erginöz, ${ }^{3}$ Türkay Demir, ${ }^{5}$ Oya Ercan. ${ }^{1}$ Istanbul University-Cerrahpaşa, Department of Pediatrics, Istanbul, Turkey; ${ }^{2}$ stanbul University-Cerrahpaşa, Department of Pediatrics, Division of Adolescent Medicine, Istanbul, Turkey; ${ }^{3}$ Istanbul University-Cerrahpaşa, Department of Child and Adolescent Mental Health and Diseases, Istanbul, Turkey; ${ }^{4}$ Istanbul UniversityCerrahpaşa, Department of Public Health, Istanbul, Turkey; ${ }^{5}$ Istanbul University-Cerrahpaşa, Department of Pediatrics, Division of Adolescent Medicine and Endocrinology, Istanbul, Turkey

\subsection{6/archdischild-2019-epa.865}

Objective The aim of this study was to determine whether there were any associations between disordered eating attitudes and behaviors and depression and anxiety among obese adolescents.

Methods A prospective cross-sectional study comprising 80 obese adolescents was performed from November 2013 to September 2014. Disordered eating attitudes were evaluated by the 'Eating Disorder Examination Questionnaire (EDE-Q)' and 'Dutch Eating Behavior Questionnaire(DEBQ)'. 'Eating Disorder Examination Questionnaire' contains four subscales that evaluate restraint, eating concerns, shape concerns, and weight concerns, and the items evaluate eating attitudes of the individual over the last four weeks. 'Dutch Eating Behavior Questionnaire' comprises three subscales which measure restrained eating, emotional eating, and external eating behaviors. Psychiatric examinations were performed for binge eating disorder (BED). Depression was evaluated by 'Children's Depression Inventory (CDI)', and anxiety was evaluated by the 'State-Trait Anxiety Inventory for Children'. Mann-Whitney U, Chi-Square and Pearson Correlation tests were used for statistical analysis.

Results Mean age of the subjects was $14.01 \pm 1.59$ years. Forty-six $(57.5 \%)$ of the subjects were girls. Mean body mass index was found as $31.29 \pm 3.06 \mathrm{~kg} / \mathrm{m}^{2}$. Twenty adolescents were found to have depression and 23 adolescents were found to have BED. There were significant associations between 